Canadian Science Publishing

Journal of Unmanned Vehicle Systems Revue des systèmes de véhicules télécommandés

\title{
Formulation of Aerial Platform Environmental Requirements from Global Radiosonde Data
}

\begin{tabular}{|r|l|}
\hline Journal: & Journal of Unmanned Vehicle Systems \\
\hline Manuscript ID & juvs-2016-0010.R1 \\
\hline Manuscript Type: & Note \\
\hline Date Submitted by the Author: & 28-Aug-2016 \\
\hline Complete List of Authors: & $\begin{array}{l}\text { Stelman, Jacob; Facebook Inc, } \\
\text { Liu, Zhang; Facebook, Connectivity Labs }\end{array}$ \\
\hline Keyword: & UAV, HAP, Aerial platform, Climate, Cloud computing \\
\hline $\begin{array}{r}\text { Please Select from this Special } \\
\text { Issues list if applicable: }\end{array}$ & \\
\hline \multicolumn{2}{|l}{} \\
\hline
\end{tabular}

SCHOLARONE ${ }^{m}$

Manuscripts 


\title{
Formulation of Aerial Platform Environmental Requirements from Global Radiosonde Data
}

\author{
Jacob Stelman and Zhang Liu \\ f Facebook Connectivity Labs \\ Woodland Hills, CA
}

\begin{abstract}
Successful operation of an aerial platform requires that it be designed with respect to the environments in which it will function. This paper demonstrates how raw observational climate data can be processed to characterize global climate histories and statistics for the formulation of aerial platform environmental requirements.
\end{abstract}

\section{Introduction}

Within a decade, High-Altitude Platforms (HAPs) will support novel civilian applications such as remote sensing, search and rescue, and Internet distribution. For instance, Facebook plans to design and trial technology to supply Internet to the underserved parts of the world by using its High Altitude Long Endurance (HALE) HAP named Aquila (Parikh, 2015). Aquila, which has a wingspan $10 \mathrm{~m}$ greater than that of a Boeing 737 and only $3 \%$ of its mass, will fly for months at a time in the mid-ranges of the stratosphere $(18-27 \mathrm{~km})$ with payloads that transmit Internet service to the ground
(Talmor et al., 2016).

To ensure the success of aerial platforms such as Aquila, design requirements need to take into consideration climatic conditions of desired operating environments. For instance, if a given aerial platform is required to be operational $99 \%$ of the time in a certain region, it must be designed to withstand the $99^{\text {th }}$ percentile of that region's extremal environmental conditions. This paper demonstrates how raw radiosonde data can be processed to characterize global climate histories to aid in the establishment of environmental requirements.

\section{Data/Methods}

NOAA's Integrated Global Radiosonde Archive V2 Beta (hereafter referred to as IGRA), a collection of 70 years of daily sounding measurements taken from balloons launched at 2700 stations worldwide (Figure 1), was chosen as the source of climate data for this analysis (Durre et al. 2006; Durre 2014). The IGRA consists of

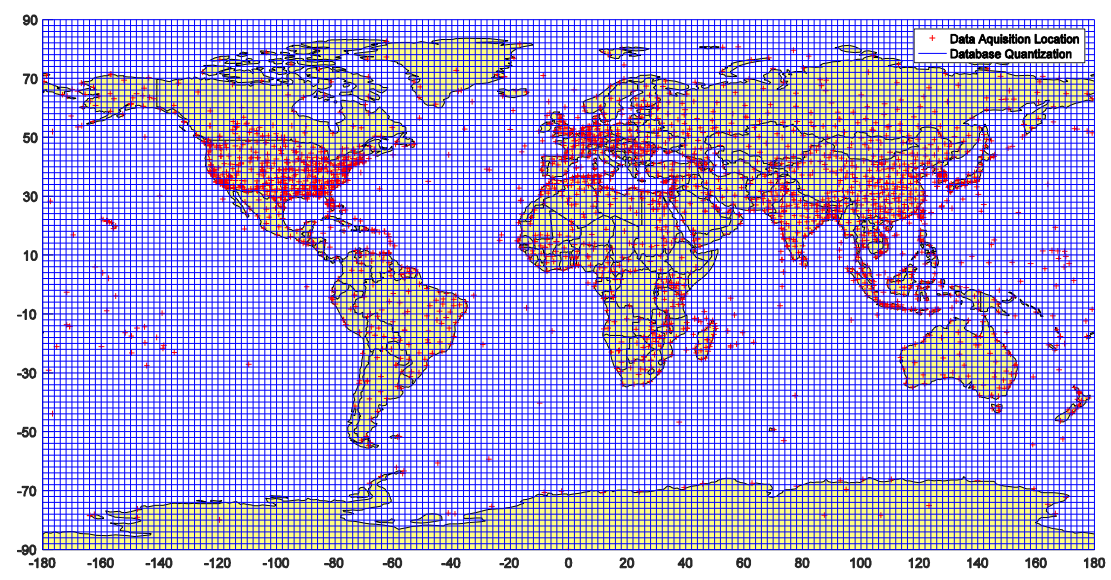

Figure 1: Data Acquisition locations of IGRA. 
roughly 80GB of measurements recorded (at various times and altitudes during a balloon's ascent) of the following quantities: wind speed, wind direction, temperature, pressure, relative humidity and dew point depression (hereafter referred to as "climate quantities") (Durre, 2014).

Parallel computing was used to grid (aggregate) the IGRA data into a central data structure for statistical climate analysis. The process begins by partitioning IGRA attributes into discrete bins representing ranges of values of interest. For example, the world was divided into two degree squares of latitude and longitude, altitude was segmented into various layers, and the values of climate quantities were separated into ranges of interest. The gridding process consists of iterating through all of the IGRA data records and recording occurrences of climate quantity measurements within established bins. The corresponding latitude, longitude, altitude, and time of the measurements, hereafter referred to as "lookup quantities", are used to "index" the occurrences into a central data structure.

The central data structure chosen for this application is a multidimensional array with dimensions representing latitude, longitude, altitude, time, the recorded climate quantities, and their respective ranges of values. An additional metadata structure is used to determine the values of bins represented by positions along the dimensions of the central data structure.
Elements in the array store occurrences of climate quantity measurements, whereas an element's position along a given dimension of the array represents the bin corresponding to a range of values symbolized by the particular dimension.

The choice of an array for the central data structure lends itself to fast statistical calculations in an array-driven language such as MATLAB or Python. For example, a Probability Density Function (PDF) of a climate quantity over a specific range of look-up quantities can be obtained by taking a "slice" of the central data structure and summing over the appropriate dimensions (therefore compounding occurrences) until one is left with a one-dimensional array. By normalizing this array, one obtains a PDF.

For ease of analysis, Geographic Information System (GIS) shapefiles were parsed to allow one to index into the central data structure based on country name. Shapefiles contain vector GIS data including country names and corresponding polygons that represent the borders of a country. One can build a key-value pair data structure to map a string containing the name of a country to an array of corresponding latitude and longitude indices representing the data recorded within the border of the country in the central data structure. This allows one to effectively specify a list of country names, and view climate statistics as is demonstrated in the following example.

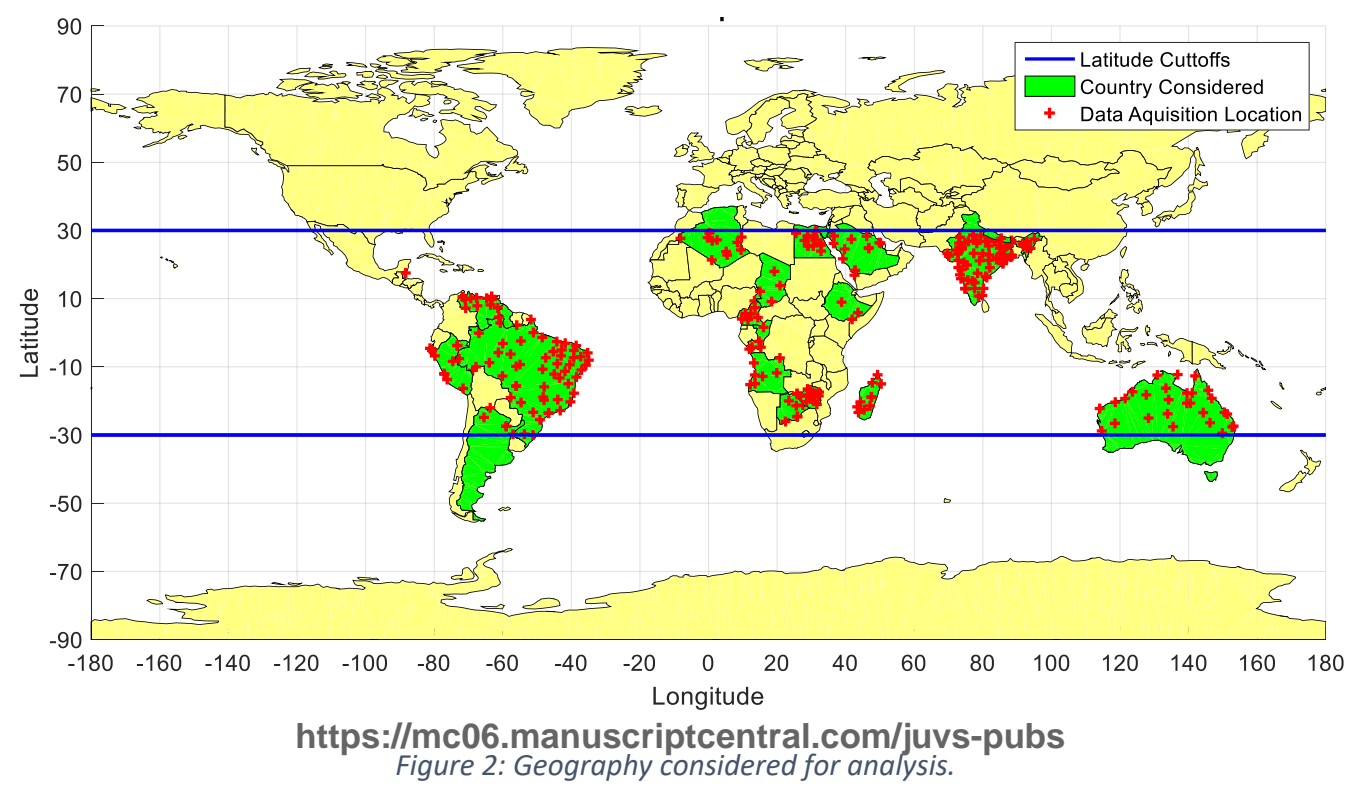




\section{Example Analysis}

The following example analysis is merely a demonstration of what can be done with this tool. The geography of interest was arbitrarily chosen, and in no way represents the regions Facebook hopes to serve with its Aquila HAP.

Suppose one wishes to determine environmental requirements for an aerial platform that would serve a latitude range of \pm 30 degrees over the following list of countries: Algeria, Angola, Argentina, Australia, Belize, Botswana, Brazil, Cameroon, Chad, Congo, Egypt, Ethiopia, Guyana, India, Madagascar, Nepal, Peru, Saudi Arabia, Venezuela, and Zimbabwe. The list of countries can be used to index into the central data structure and extract climate information (Figure 2).

Different aerial platforms will be sensitive to different environmental conditions and therefore different climatic data will be pertinent. What follows is a general overview of the data that can be obtained, and its application to a general aerial platform.

Computing the PDF of wind direction for various altitude ranges yields the following plot:

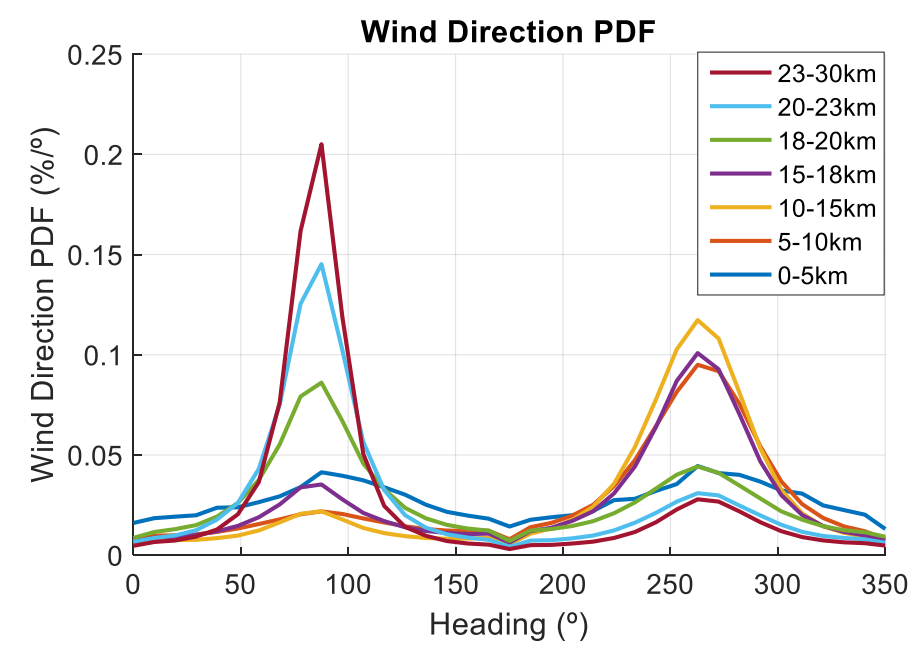

Figure 3: PDF of Wind Direction at various altitude ranges.

One can see from Figure 3 that at lower altitudes the wind blows predominantly from east-to-west, and from west-to-east at higher altitudes. Balloon platforms can exploit this wind behavior to station keep by controlling their altitude to catch wind in different directions.

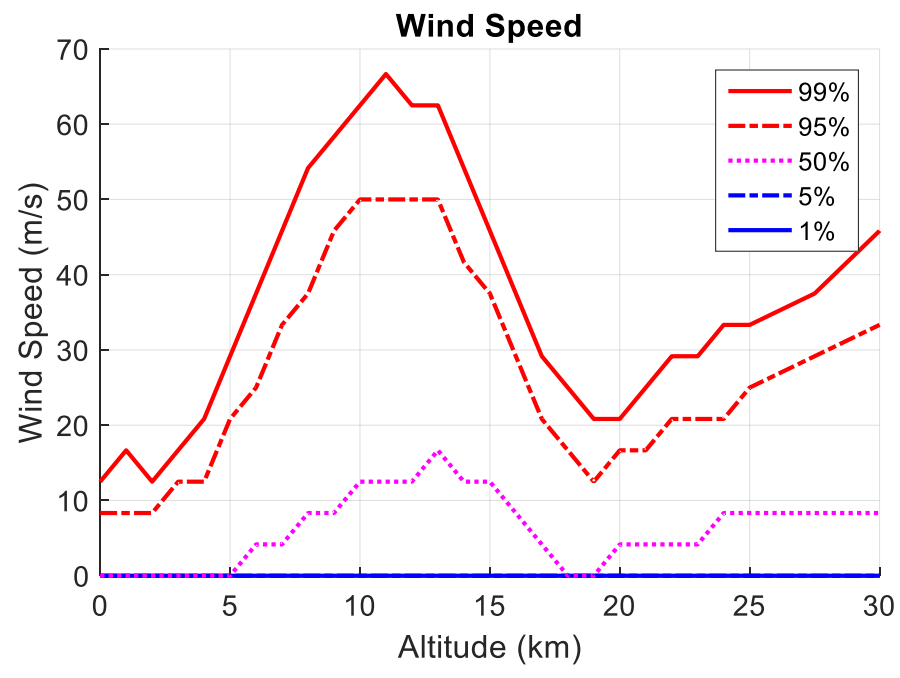

Figure 4: Wind speed percentiles vs altitude.

Computing percentiles of wind speed at various altitudes (Figure 4) shows that large wind magnitudes exist in the upper-troposphere $(10 \mathrm{~km})$ through lower-stratosphere $(15 \mathrm{~km})$ whereas in the mid-stratosphere $(20 \mathrm{~km})$ there is a lull in wind speed. This is a suitable operating altitude for aerial platforms since station keeping at a geographic location requires flying against the wind.

Thermal requirements can be challenging at high altitudes since low air density makes heat rejection difficult. A plot of temperature percentiles vs altitude can be used for thermal analyses. Similar statistics on temperature can be obtained from MIL-HDBK-310 ("Global climatic data for developing military products", 1997), but it is important to note that the statistics in the mil handbook are for the entire earth and do not tailor to specific regions of interest.

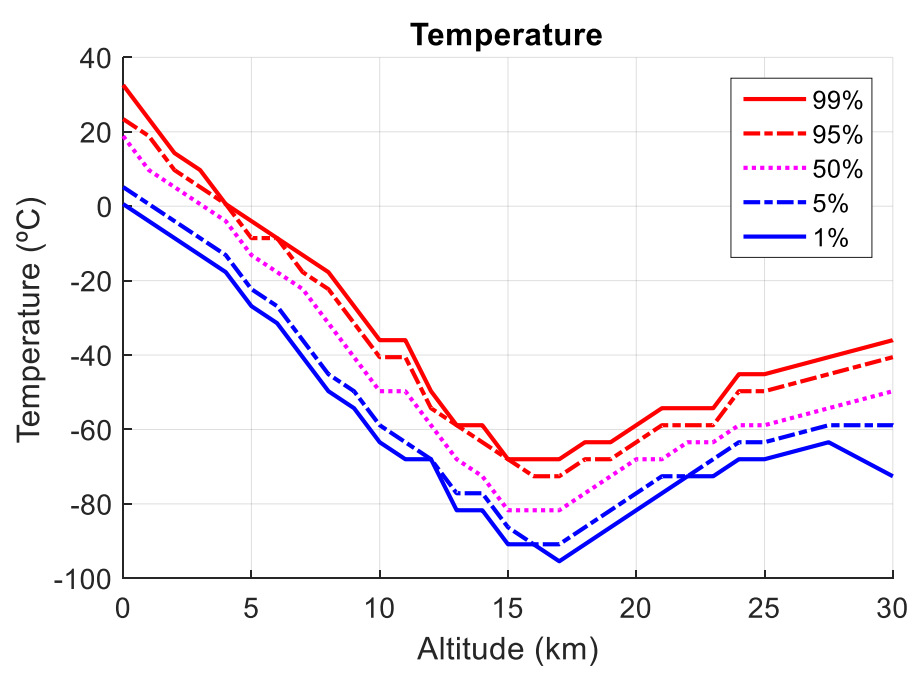

Figure 5: Temperature percentiles vs altitude. 
Figure 5 shows that an aerial platform operating at $20 \mathrm{~km}$ must be able to withstand temperature fluctuations ranging from $-60^{\circ} \mathrm{C}$ to $-80^{\circ} \mathrm{C}$.

Relative humidity data (Figure 6) can be combined with pressure data (Figure 7) and temperature data (Figure 5) to determine air density at altitude. Air density has major effects on lift, drag, and overall performance of an aircraft. Moisture in the air causes air density to decrease and therefore reduces lift and increases power requirements; although this effect is minimal unless one is at high temperature and high relative humidity in a low altitude.

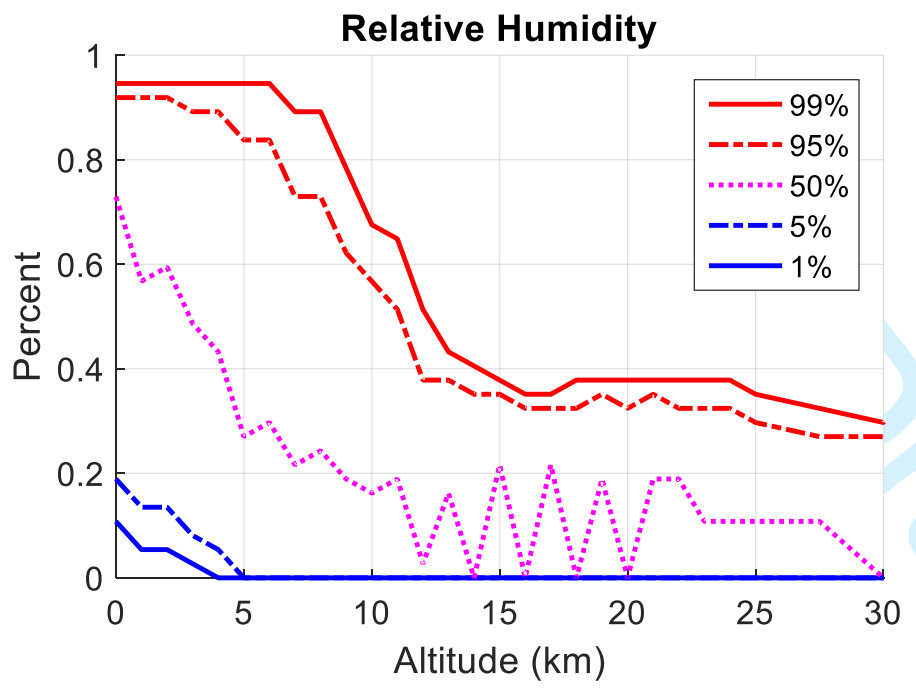

Figure 6: Relative humidity percentiles vs altitude.

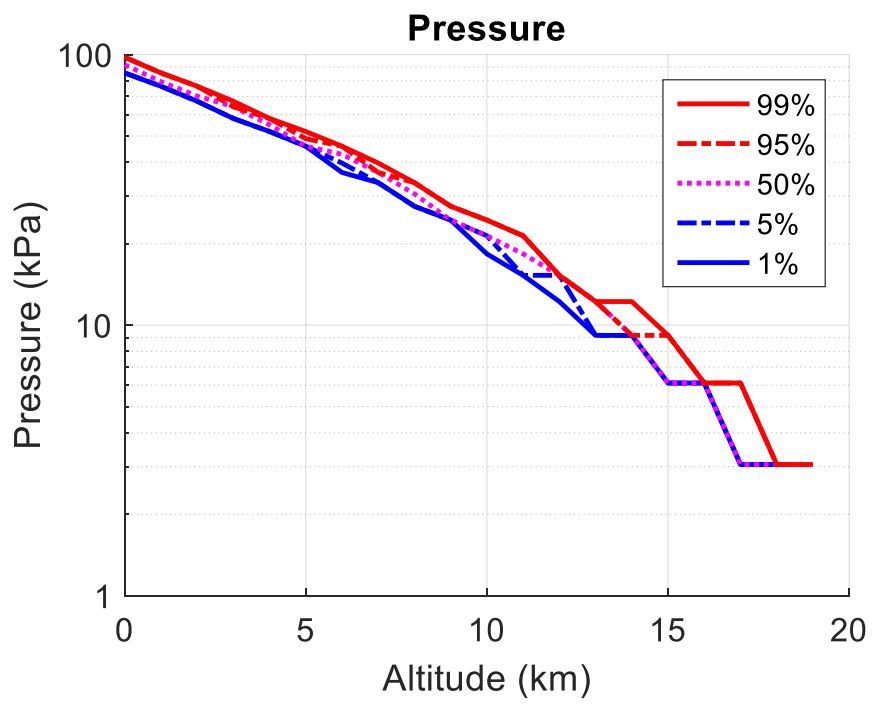

Figure 7: Pressure percentiles vs altitude.

If an aerial platform were equipped with optical payloads, cloud presence in operating environments may be relevant. Although full discussion is beyond the scope of this paper, relative humidity can additionally indicate the likelihood of cloud formation (van Heerwaarden and Guerau de Arellano, 2008; Haag et al., 2003). This is problematic since cloud cover can greatly retard the performance of a Free-Space Optical (FSO) communication payload (Hemmati, 2009).

\section{Limitations}

The central data structure used in this analysis is very sparse because the IGRA does not contain data for every point on the earth. At this time, neither MATLAB nor Python/SciPy support sparse arrays greater than two dimensions ("Matlab sparse", 2016; "Sparse matrices", 2016). Since the central data structure cannot be stored in a sparse fashion, memory must be allocated for every element it contains, which limits the possible number of elements to something that can fit into a typical personal computer's RAM. This reduces the possible resolution of the discrete partitions since finer resolutions require more elements and therefore more memory.

By finding a method to store the central data structure in a sparse manner, or by changing the central data structure, finer resolutions of climate data could be stored and analyzed.

\section{Conclusion}

This paper presents a general method for characterizing the climate history of a given region to aid in the establishment of aerial platform environmental requirements.

The method presented is not limited to the IGRA climate data processing, it may be applied to other observational climate datasets such as NASA's Moderate Resolution Imaging Spectroradiometer (MODIS), Atmospheric Infrared Sounder (AIRS), Tropical Rainfall Measuring Mission (TRMM), etc (Crichton et al., 2012).

\section{Acknowledgments}

We wish to thank our colleague, ChienChung Chen, for his initial analyses of IGRA data that inspired the formulation of this method. 
We additionally thank Paul Ullrich of the University of California, Davis for his technical feedback on this paper, and Mathew Oliver of the University of California, Davis for his stylistic suggestions. 


\section{References}

Crichton, D., Mattmann, C., Cinquini, L., Braverman, A., Waliser, D., Gunson, M., Hart, A., Goodale, C., Lean, P., Kim, J. 2012. Sharing satellite observations with the climate-modeling community: software and architecture. IEEE Software. 29(5): 73-81.

doi:10.1109/ms.2012.21.

Durre, I. 2014. Integrated Global Radiosonde Archive (IGRA) V2 beta readme file [online]. National Climatic Data Center. Available from http://www1.ncdc.noaa.gov/pub/data/igra/v2beta/igra2readme.txt [accessed 13 March 2016].

Durre, I., Vose, R., Wuertz, D. 2006. Overview of the Integrated Global Radiosonde Archive. Journal of Climate. 19(1): 53-68. doi:10.1175/jcli3594.1.

Global climatic data for developing military products. 1997. United States Department of Defense. MILHDBK-310.

Haag, W., Kärcher, B., Ström, J., Minikin, A., Lohmann, U., Ovarlez, J., Stohl, A. 2003. Freezing thresholds and cirrus cloud formation mechanisms inferred from in situ measurements of relative humidity. Atmos. Chem. and Phys. 3(5): 1791-1806. doi:10.5194/acp-3-1791-2003.

Hemmati, H. 2009. Near-earth laser communications. CRC Press. Boca Raton, U.S.

Parikh, J. 2015. New milestones in Connectivity Lab's aircraft and laser programs [online]. Facebook Newsroom. Available from http://newsroom.fb.com/news/2015/07/new-milestonesin-connectivity-labs-aircraft-and-laser-programs/ [accessed 13 March 2016].

Talmor, A., Harding, H., and Chen, C. 2016. Two-axis gimbal for air-to-air and air-to-ground laser communications [online]. Research at Facebook. Available from https://research.facebook.com/publications/two-axisgimbal-for-air-to-air-and-air-to-ground-lasercommunications/ [accessed 13 March 2016].

van Heerwaarden, C., Guerau de Arellano, J. 2008. Relative humidity as an indicator for cloud formation over heterogeneous land surfaces. J. Atmos. Sci. 65(10): 3263-3277. doi: 10.1175/2008jas2591.1.

MATLAB sparse [online]. 2016. Mathworks. Available from http://www.mathworks.com/help/matlab/ref/sparse.html [accessed 13 March 2016].
Sparse matrices [online]. 2016. SciPy Reference Guide. Available from http://docs.scipy.org/doc/scipy/reference/sparse.html [accessed 13 March 2016]. 